\title{
MOBILE LASER SCANNING IN HIGHLY URBANIZED AREA - A CASE STUDY IN SOFIA
}

\author{
C. Mickrenska-Cherneva ${ }^{1 *}$, A. Alexandrov ${ }^{2}$ \\ ${ }^{1}$ University of Architecture, Civil Engineering and Geodesy, Geodesy and Geoinformatics Department, Sofia, Bulgaria \\ kmikrenska_fgs@uacg.bg \\ 2 Patishta LTD, Plovdiv, alexanderdalexandrov@gmail.com
}

\section{Commission IV}

KEY WORDS: Cadastre, Total Station, Mobile Laser Scanning, 3D Modelling, Quality control

\begin{abstract}
:
The paper examines the possibility to create a three-dimensional model of cadastral objects - cadastral parcels and buildings, exploring different spatial data acquisition technologies. Four cadastral parcel and part of the buildings situated in them are selected for the range of the task. Self-contained objects located in buildings have not been examined. The objects are located on the territory of Lozenets District, Sofia, which is highly urbanized part of the capital. In order to create a three-dimensional model, classical land surveying measurements with a total station and mobile laser scanning were performed.

In addition, the acquired data is assessed. It is made by comparing measurements of the building to the measurements of the registered point cloud. The assessment results show that the mobile laser scanning technology is efficient to reproduce high quality and accurate data for existing building structures due to MLS advantages - the large range, high accuracy, fast data acquisition and high level of detail.
\end{abstract}

The paper is a part of Alexander Alexandrov master thesis.

\section{INTRODUCTION}

For the purpose of the examination are made classical land surveying measurements with a total station and mobile laser scanning. The processes of data acquisition and processing are compared. The distance measurements by a measuring tape are made in order to control the quality of two created models.

The surveying measurements with a total station is well-known technology used since the late 1960s. It is used for 3D coordinates determination of measured points and creation of various 3D models.

Laser Scanning technology have been developed since the end of 1990s for 3D digital measurement, documentation, modelling and visualization. Laser Scanning is a process of using a line of laser light in order to capture digitally the shape, size and colour of objects. The application areas are growing nearly every day, because of the advantage of laser scanning - the amount of data that can be captured with a high accuracy in a relatively short period of time. The collected data can be used to construct digital 2-dimensional (2D) drawings and plans or 3dimensional (3D) models.

\section{USED TECHNOLOGIES FOR GEOSPATIAL DATA COLLECION}

Ground control points are required for the collection of any geospatial data, as the final results must be in a common coordinate system or at least a rapid and reliable transformation from one coordinate system to another is possible [Ivanov R., 2020].

The main geospatial information acquisition methods used are classical methods (total stations), GNSS technologies, photogrammetric technologies, and laser scanning. In order to make any measurements, control points are needed from which to proceed. Some of these methods require more preparation, others not so much [Alexandrov B, 2012].
- Classical method (by total station) - For coordinate determination of a detailed point is necessary to be measured a direction and distance. It is required a lot of known control points, which in many cases is not sufficient to be performed measurements with a total station. In order to measure the necessary details in most cases, it is necessary to coordinate additional points that should be preliminarily determined. The method is time consuming, but the accuracy of the measured points is high. Requires a team of at least two people. The detailed points that are measured are just what we need, without many stray points, and consequently the post-processing is easier. But it is imperative that the points that are shot be walked precisely for the sake of convenience. Another option to keep track of measurements during measurements is to use a "coded surveying", which in turn requires rigorous organization and timely coding of the surveyed points during field activities [Pavlov P., 1999].

- GNSS technologies - For the design and coordination of ground control points, they are the most used method, but when it comes to collecting 3D spatial information, this method alone is not very appropriate. When it is used in combination with one of the others, the work is optimized. Not suitable because there are many inaccessible points that the operator cannot reach, and the range in densely urban areas sometimes is a problem.

- Photogrammetric technologies - photogrammetry is used to gather aerial or terrestrial spatial information, with varieties having their advantages and disadvantages.

- Terrestrial laser scanning - The terrestrial laser scanner measures non-reflective distances from the scanner to surrounding objects by emitting pulses in different directions and is received by the photodetector of the reflected from the measured object. Depending on the measurement technology used, they are divided into phase and pulse [Antova G., 2006]. According to Bulgarian legislation, allowable methods for geospatial data acquisition total stations, GNSS technologies, 
photogrammetric technologies and combinations of these three methods.

\section{LASER SCANNING TECHNOLOGY}

Because the other methods have long been well-known, only the laser scanning technology will be presented in brief.

Terrestrial Laser Scanning (TLS) is defined as "the use of a ground based device that uses a laser to measure the 3Dcoordinates of a given region of an object surface automatically, in a systematic order at a high rate in near real time" [Mills and Barber, 2003].

The result will be a group of points with particular properties, called point cloud. It is specifically defined as "a collection of $\mathrm{XYZ}$ coordinates in a common reference system that portrays to the viewer an understanding of the spatial distribution of a subject or site" [Mills and Barber, 2003].

The terrestrial laser scanner could be set on a stationary station or be mounted on a movable device. Depending on the application field, measured ranges vary from centimetres to thousands of meters. This point cloud will need a number of processing steps and improving in which it becomes sufficient for using in any specific application. Processing of point clouds is achieved through a software package in which specific results are obtained from the scanned points [Dzhenkova R., 2019].

Nowadays, there is a wide range of $3 \mathrm{D}$ laser scanning processing software packages on the market, which use different methods and approaches in processing the scanned data. The data displayed in a form of point cloud is very useful as it is able to be presented in many ways.

In other words, 3D laser scanning is a way to capture digitally the object's size, shape and colour into the computer world as a $3 \mathrm{D}$ representation.

The terrestrial laser scanner can be described as a motorized total station, which measures automatically all the points in its horizontal and vertical field. The application area is growing nearly every day, because of the advantage of laser scanning the amount of data that can be captured with a high accuracy in a relatively short period of time.

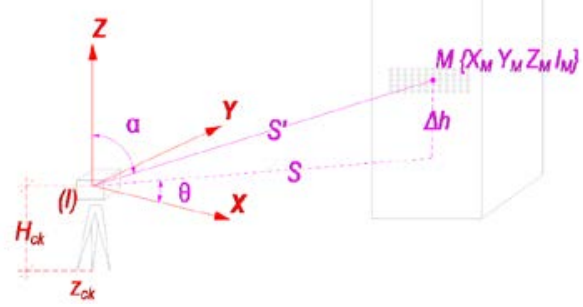

Figure 1. Coordinate positioning of scanned points

Each point is determined by its spatial coordinates $\mathrm{X}, \mathrm{Y}, \mathrm{Z}$ and the intensity of the reflected signal $l$. The terrestrial laser scanner sends laser pulses to the object and receives the reflected signal (figure 1). The coordinate system of the scanner for the coordinates of each scanned point $\mathrm{M}$ is:

$$
\begin{aligned}
& X_{M}=X_{C K}+S^{\prime} \cos \theta \cos \alpha \\
& Y_{M}=Y_{C K}+S^{\prime} \cos \theta \sin \alpha \\
& Z_{M}=Z_{C K}+H_{C K}+S^{\prime}{ }^{\prime} \operatorname{ctg} \alpha
\end{aligned}
$$

where: $X_{C K}, Y_{C K}, Z_{C K}$ are the coordinates of the scanner postition;

point;$$
H_{C K} \text { - the height of the scanner head from the groun }
$$

$$
S \text { '- slope distance from scanner head to scan point; }
$$

$\alpha$ and $\theta$ - polar angles determining the position of the scanning beam;

$$
X_{M}, Y_{M}, Z_{M} \text { - coordinates of the scanned point } \mathrm{M} \text {; }
$$$$
l \text { - the intensity of the reflected signal from point } \mathrm{M} \text {; }
$$

\subsection{Classifications}

According to range, the scanning systems can be divided into different groups - on the object of importance (from small objects to large buildings), with a wide range of scales (from few mm up to tens of hundreds meters):

- Airborne Laser Scanning (ALS) - such a system is used on an aircraft to capture 3D-data of large areas, such as urban and industrial areas, agricultural or forestry sites, etc.

- Terrestrial Laser Scanning (TLS) - a ground-based version of the Airborne Laser Scanning used mainly for terrain and landscape mapping, buildings, designing and construction of roadways and bridges, etc.

- Micro-Laser scanning (MicroLS) - such a system is used to scan an object over a short distance (from $\mathrm{mm}$ to a few metres) and is mainly applied to reverse engineering and prototyping, quality control/inspection and documentation of cultural artefacts, etc.

The terrestrial laser scanners that are being used for topographic mapping and modelling can be operated in two modes - static (stationary) or dynamic (moving).

- Static laser scanning - the scanner is positioned in a fixed position during the data acquisition. The advantages of this method are the high accuracy and its relatively high point density. Generally, the scanner is being mounted on a tripod over a known control point.

- Dynamic laser scanning - the scanner is mounted on a mobile platform and is in motion during measurement. These systems require additional positioning systems such as GNSS and Inertial Navigation System (INS). Dynamic laser scanners are used on mobile mapping vehicles and manned or unmanned aircraft.

\subsection{Classification of terrestrial laser scanners}

There are wide variety of laser scanners and they have different specifications for particular applications. The classification of TLS can be done based on:

- Principle of the distance measurement system.

- Laser beam deflection unit and angle measurement system.

3.2.1 Classification based on the principle of the distance measurement system

The distance measurement system corresponds both to range and accuracy. Almost all of the terrestrial laser scanners use mainly three methods of scanning:

1) Pulse-based - the pulse scanners use the Time-offlight (TOF) between emitted and received beam pulse. The pulse-based scanner uses laser light to detect the target.

2) Phase-based - the phase scanners compute ranges by measuring the difference in the wave phase between emitted and received beam. Phase-based terrestrial laser scanning has a high scanning speed and good accuracy rate.

3) Triangulation-based - the triangulation laser scanners shine a laser dot on the object and use a camera to look for the location of the laser dot. A triangulation-based laser scanner is commonly used in capturing small or medium size object such as ancient items, artefacts and antiques.

3.2.2 Classification based on laser beam deflection and angle measurement system

An important characteristic of terrestrial laser scanners is the Field-of-view (FOV), which is determined by the beam 
deflection unit of the scanner. A 2D-deflection unit enables imaging in horizontal and in vertical. This deflection unit combined with a laser measurement system enables to determine 3D-coordinates of the object of interest.

There are three types of beam deflection units used in TLS: oscillating mirrors, rotating polygonal mirrors and monogon rotating mirrors which represents the FOV of the TLS.

The different scanner types based on FOV are camera scanners, hybrid scanners and panorama scanners.

- A camera field of view is achieved by using two synchronized mirrors - one for horizontal beam deflection and one for vertical beam deflection [Fröhlich and Mettenleiter, 2004]. The scanning head remains stationary during scanning process.

This type of scanning is well suited to the survey of a specific site or a building facade. Camera view systems are usually used with pulsed laser technology.

- Hybrid scanners are scanners where one rotation axis (often the horizontal movement) is without restrictions, while the second rotation axis uses oscillating mirrors. These mirrors are comparatively slow and provide limited vertical field-of-view (maximum $90^{\circ}$ ).

This type of scanning is carried out when there is no requirement to measure objects overhead or at steep vertical angles. These scanners are typically used in pulsed laser scanners.

- A panoramic view is achieved by a single rotating (azimuth deflection) mirror and simultaneously rotating the system by its centre axis.

\subsection{Non-instrumental error}

The accuracy of the measurements obtained from terrestrial laser scanners also depends on many non-instrumental factors:

- angle of incidence;

- surface properties (reflectivity);

- surface properties (colour, roughness, moisture);

- surface properties (edge);

- environmental errors.

\subsection{Mobile laser scanning}

Compared to traditional capturing methods that use digital imaging or aerial laser scanning systems, the accuracy of obtained data by mobile laser scanning is much better. In addition, measurement time and cost are reduced compared to total station measurement. Mobile Laser Scanning (MLS) is more efficient than terrestrial in terms of data collection or cloud point density. It is characterized by high accuracy $(2 \div 10$ $\mathrm{mm}$ ), high resolution ( $3 \div 15 \mathrm{~mm}$ dot spacing) and low noise.

The mobile laser scanning system provides instantaneous localization of the observer in the coordinate system, defining such a reference and appropriate time frame. Also, all acquired environmental data need to be synchronized in the same timeframe (epoch) for their proper georeferencing.

MLS is usually a combination of GNSS technology and an inertial measurement unit (IMU) for complete georeferencing. GNSS positioning alone is a method in which results may be erroneous due to the lack of visibility of satellites, a condition that is common in urban environments or in high vegetation. To overcome this and provide information on the position of the orientation sensor to the mapping system, IMU equipment is also included.

The IMU provides high frequency data and its location and position by monitoring changes along and around the three axes of linear acceleration and angular velocity. Inertial navigation provides the necessary positioning accuracy for short period of time; in larger ones, the solution is mistaken for gyro deviation and accelerometer error. These errors are corrected through GNSS positioning, but in the absence of a satellite signal for a longer period of time, the errors will gradually become large.

\subsubsection{Mobile Laser Scanning Errors}

MLS is a combination of different systems and sensors. At the same time, the GNSS provides accurate location information for the vehicle, while the IMU provides information on the spatial position of the vehicle. Measurements using the MLS method are fraught with various errors that affect the accuracy of the measurements [COŞARCĂ et al, 2009].

MLS errors can be classified in different ways:

- From sensors (GNSS errors, IMU, instrument errors)

- From the scanned object (Reflectivity and signal multipath)

- From the environment (Temperature, atmosphere, interfering radiation, distraction from movement)

- From the integration of different systems (Errors from installation and time synchronization)

- Methodological errors.

\section{A CASE STUDY OF 3D MODELLING IN HIGHLY URBANIZED AREA}

\subsection{The range of case study}

The range of case study are four cadastral parcels with identifiers 68134.902.236, 68134.902.235, 68134.902.248, 68134.902.714 and the part of buildings situated in them as shown on figure 6 with red colour. The self-contained objects are not included in that study, because it is necessary to be done additional measurements inside the buildings.

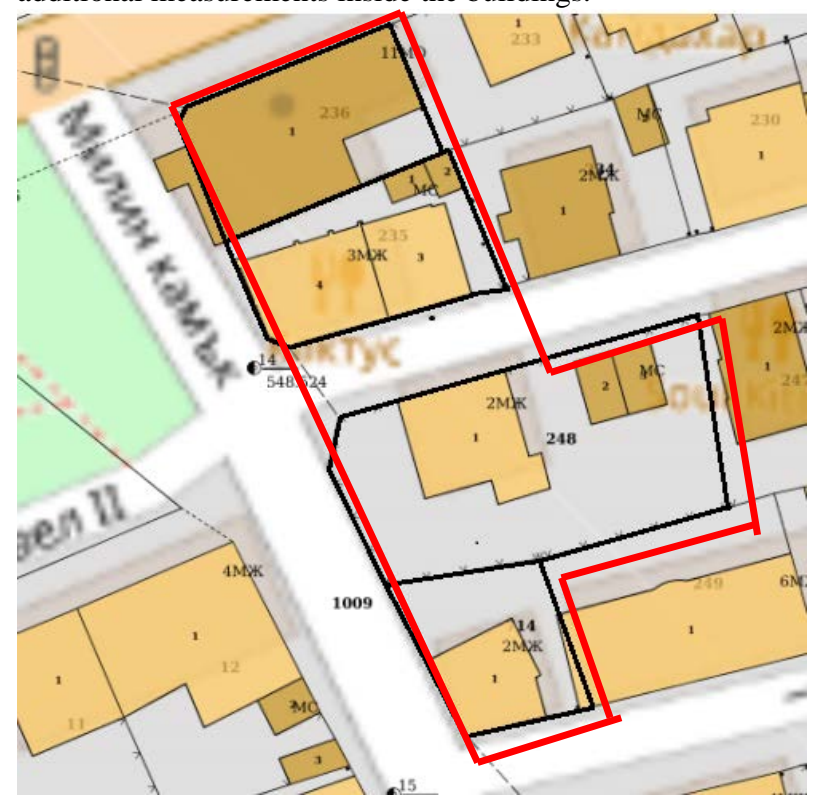

Figure 6: The range of case study

The functional purpose of the building in a parcel 68134.902.236 (Figure 7) is an office building. It consists of eleven floors. Its facade is curved, made of glass. 


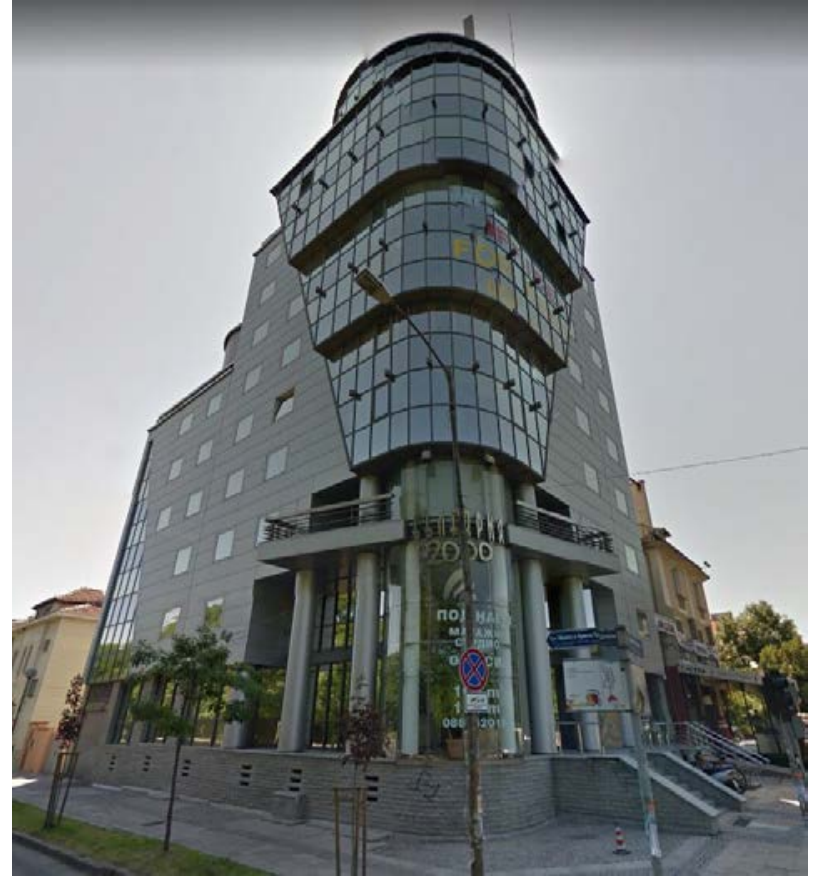

Figure 7: The building in a parcel 68134.902.236

In the next parcel 68134.902.235 is measured building 4 (Figure 8) with the functional purpose "residential building multifamily". At the first floor is situated a restaurant.

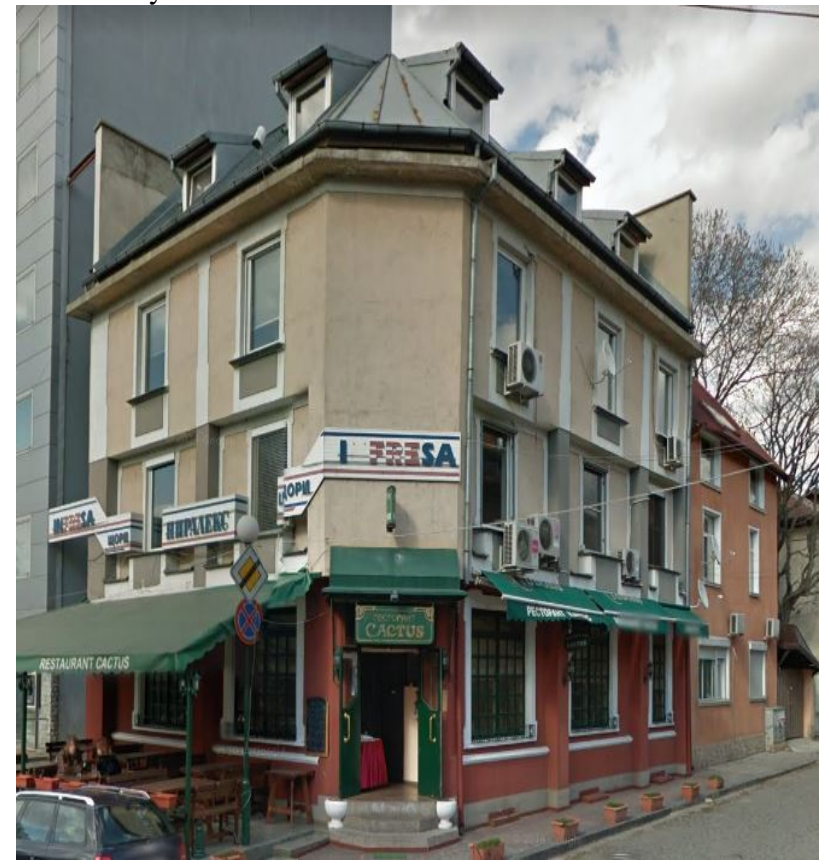

Figure 8: The buildings 3 and 4 in a parcel 68134.902.235

In parcels 68134.902 .248 and 68134.902 .714 are measured two residential buildings.

\subsection{Survey on existing control network}

Eight points from the Geodesy, Cartography and Cadastre Agency (GCCA) are found. It has been established that these points are reserved and can be used for the land surveying measurements of the buildings.

\subsection{Creation a 3D model with a total station}

The activities required to measure the buildings were carried out by one team. The team includes an operator and a person involved. A total station Leica TCR 805 was used. A telescopic pole with a Leica GPH1 prism was used.

The measurements were done by 5 points. It was stationed at 4 known (control) points (points 5, 14,15 and 3) and 1 additional new point 1.

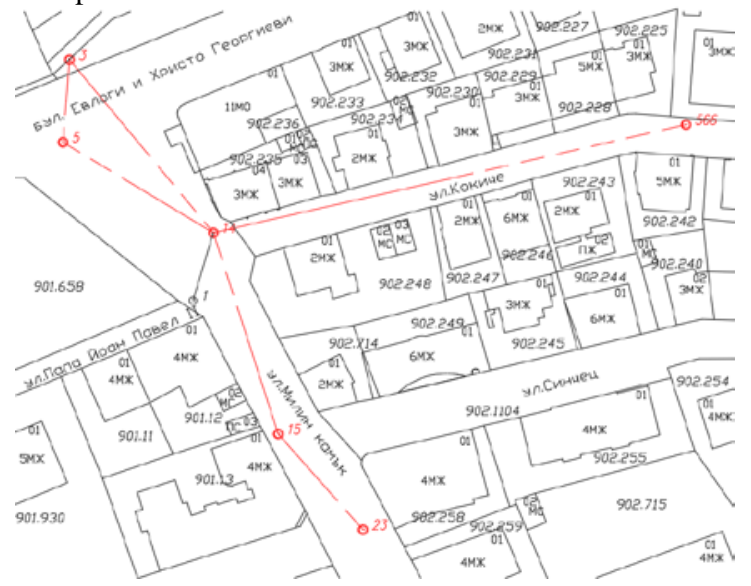

Figure 11: Location of the control points

Detailed points of the boundaries of the parcels and buildings were measured and architectural elements on the facades such as windows, doors, columns, etc., which are necessary for creation of 3D model. The accessible parts of the contours of the buildings are measured by a prism. Non-reflective measurement was used in the inaccessible parts of the facades of the building.

During the measurements, control distances were measured on buildings such as window width, plinth height, and more. At Figure 12 are shown part of them.

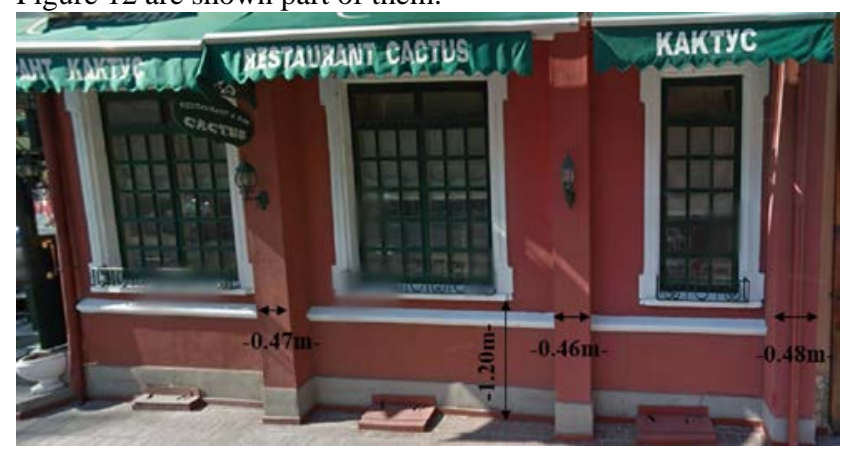

Figure 12: Measured control distances

\subsection{Creation of a 3D model by total station}

Creation of a 3D model is made by AutoDesk Civil 3D. The results are shown at figures $13,14,15$ and 16. 


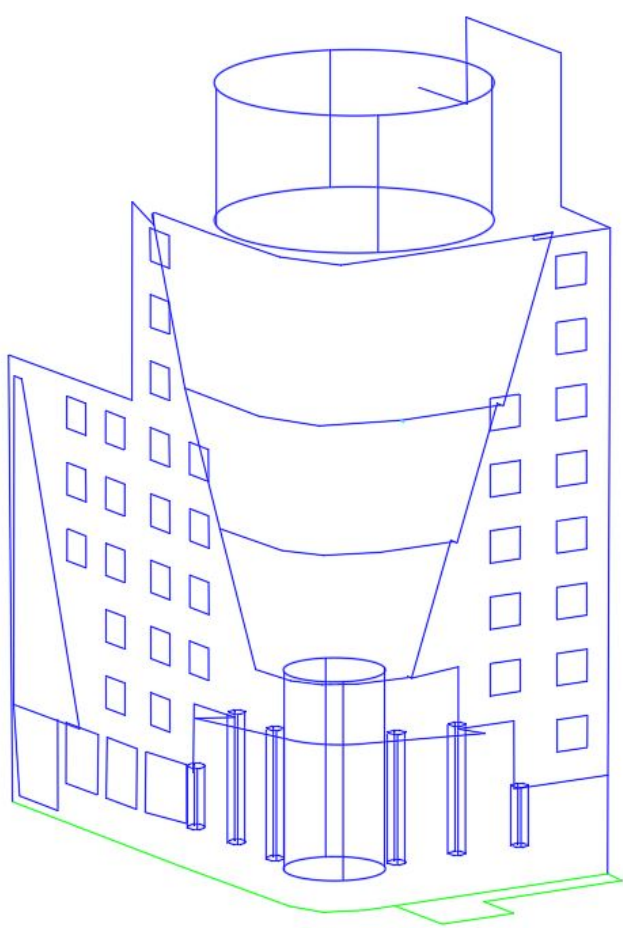

Figure 13: The building in a parcel 68134.902.236

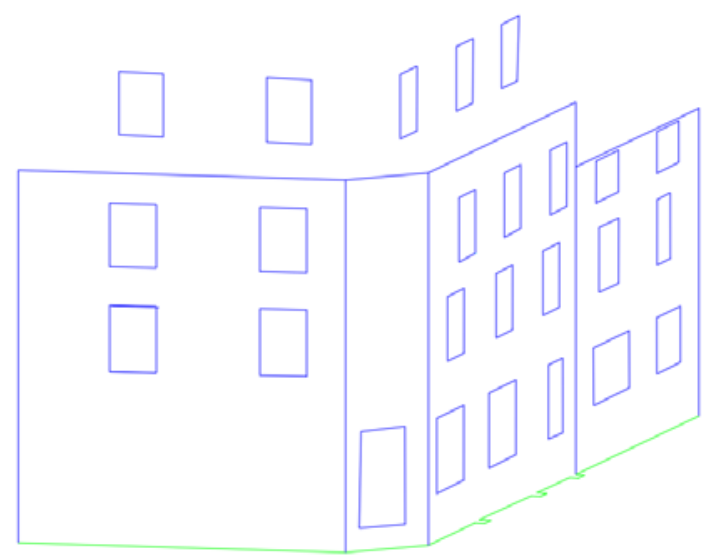

Figure 14: The buildings 3 and 4 in a parcel 68134.902.235

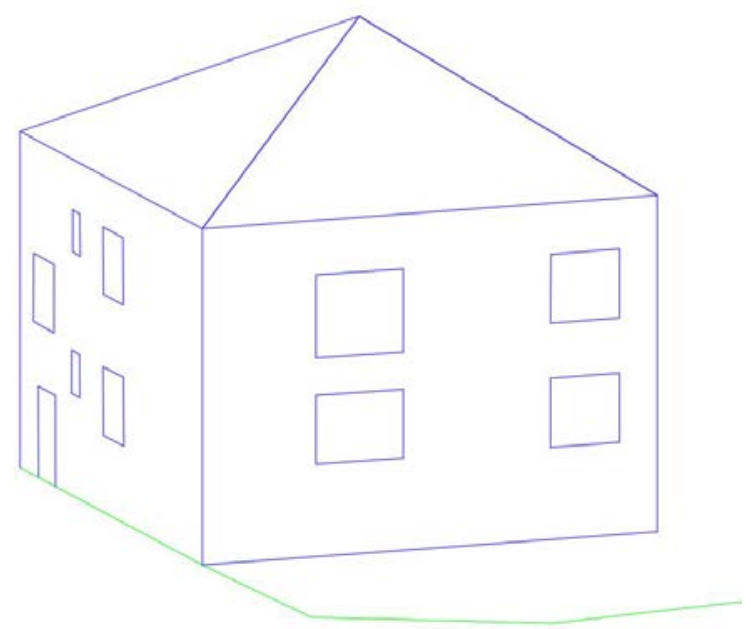

Figure 15: The building in a parcel 68134.902.248

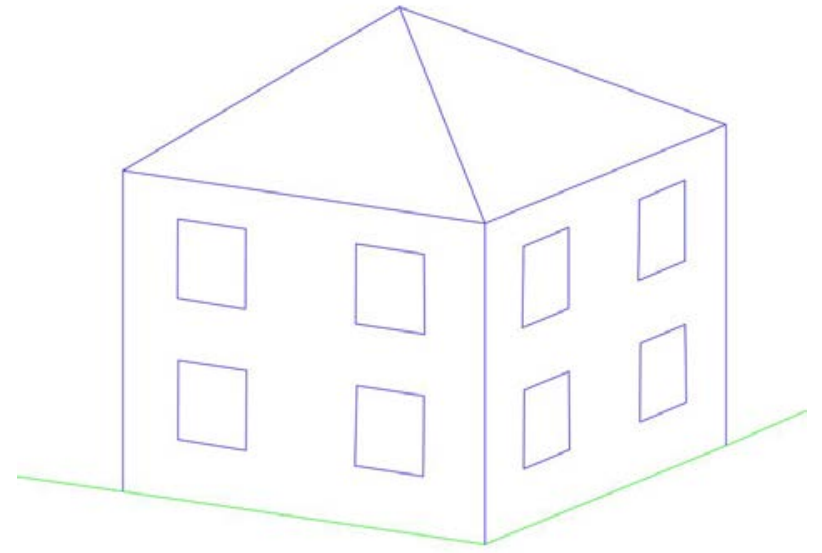

Figure 16: The building in a parcel 68134.902.714

\subsection{Creation a 3D model by mobile laser scanner}

3D Laser Scanner Riegl VMZ-2000i has been used for the purpose of the study. It features an extremely high effective measurement speed, delivering up to 400,000 measurements per second and up to 240 scan lines per second. With this instrument, long distances of more than $2000 \mathrm{~m}$ to natural surfaces can be measured while being completely eye-safe (Laser Class 1).

The used laser scanner is a compact and lightweight measuring device. It can be mounted in several positions - upright, lying down and tilted. The scanner can also operate as a stationary device in confined spaces.

Before the scan is started, the GNSS receiver is placed on a known control point, adjusting to measure in static mode. This allows better precision in the trajectory calculation.

Scanner has to be mounted on the vehicle. The installation takes about 20 minutes. The scanner is turned on and the required field data acquisition software is loaded.

\subsection{Used software for data acquisition}

Different software have been used for data collection during the scanning process.

LV POS View is a positioning and orientation system. Based on inertial technology, the system provides continuous and accurate vehicle location information, even in areas where the GNSS coverage is poor or absent.

RiACQUIRE is a major project processing software for RIEGL mobile and aerial laser scanning systems. The system consists of at least one laser scanner, a position measurement system and an operator workstation. Additional laser scanners, a camera, mass storage devices and various mechanical elements can be added to the system.

RiACQUIRE provides visual information on specific measurements from INS/GNSS systems to easily verify the plausibility of the results.

The scanning process requires two operators. One controls the scanning system and the other controls the vehicle.

Based on the attempts made, it was concluded that speeds of up to $30 \mathrm{~km} / \mathrm{h}$ are best suited for travel. At higher speeds, the appearance of "white spots" is drawn, or the images are rendered with poor quality.

To ensure that the control marks located on the site will be scanned as speed passes, the speed will be reduced.

The subject is captured three times (twice in the horizontal position of the device and once in the vertical position) to obtain the most accurate and detailed model of the subject. 


\subsection{Data processing}

The scan results represent two separate components. One is point clouds and the other is trajectories. The initial trajectory obtained from the IMU/GPS system is accurate, which is achieved by differential GNSS methods.

With the help of specialized software POSPac MMS goes to the subsequent processing of the trajectory. It boils down to calculating the desired trajectory in real time and then georeferencing to the point selected for the base station and aligned to centimetre accuracy.

After processing the trajectories, the next step is point cloud processing. The processing, management, analysis and visualization of data acquired by the Mobile Laser Scanning System (MLS) RIEGL is performed using RiPROCESS software.

The scanner data is converted to a trajectory reference system, WGS84, ITRS or ETRS using the RiWORLD program. Thus, RiWORLD retrieves the acquired data from the object surfaces in a geocentric coordinate system for further processing. The software applies detailed descriptions of the geometry system, transformations from the scanner coordinate system to the INS/GNSS coordinate system, and vehicle coordinate system to transform high precision scanner data. The input data obtained from the system is a cloud of points in the coordinate system of the trajectory with additional description for each point, such as echo amplitude, reflection, pulse width, pre-classification and others.

The next step is RiPROCESS program. It must first select a reference coordinate system for further processing. The trajectory is processed in WGS84-Geocentric and is selected in RiPROCESS.

After the reference coordinate system has been selected, some parameters are set for output. These parameters reduce the data to necessary. The range of data we receive may be differentiated from laser range, deviation, laser amplitude, reflection, etc.

For the case study purpose, the laser range is chosen to be at least $3.5 \mathrm{~m}$, since this is the minimum distance that the scanner will not take into account data from the surface of the vehicle.

An important role in the processing of mobile laser scanning data is taken part by the RiPRECISION function. It automatically corrects GNSS/INS trajectories. These adjustments are necessary in order to merge overlapping records.

RiPRECISION allows scan data to be assigned to specific control points. This results in a constant cloud of points with increased precision and increased georeferencing accuracy.

When processing each of the trajectories individually with all overlapping track records, the achieved height accuracy is $0.13 \mathrm{~m}$.

To improve system calibration, the relative alignment of scan data to minimize discrepancies between different datasets and/or the absolute adjustment of scan data with respect to the local/global coordinate system uses the Scan Data Adjustment tool.

The number of free parameters depends on whether we want to use some of the scanner rotation angles (Roll, Pitch and Yaw), or the position of the trajectory with respect to any of the three output axes.

It is most appropriate to adjust the data by not selecting any of these parameters for output (free adjustment), and then the already obtained data are adjusted with the control marks taken as output.

The similarity in the planes is determined by the set detection parameters, which are Minimum angle of inclination of the plane, Maximum deviation between points in the plane, Minimum number of points in the plane. They also make a compliance parameters for matching between different search radii, angular tolerance and maximum distance.

For the case study, 395 such (Number of observations) were detected by the Scan Data Adjustment tool.

After adjustment, results for a standard deviation of $2 \mathrm{~cm}$ are obtained by similar planes.

The point cloud must be classified to make it easier to handle. This is done by removing excess point clouds and separating them into different layers according to their properties. Vegetation, small objects, moving vehicles, distant objects, etc. are separated by a field filter. In the filtering process, the vertical distances of the points from the estimated earth surface are analyzed. Based on these distances, points are classified on or off the ground.

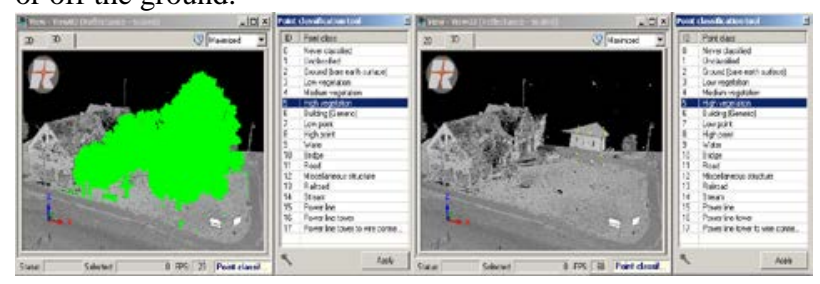

Figure 17: Point cloud after the high vegetation point classification process

Data export is done in an appropriate coordinate system. When creating a project in RiPROCESS, it is automatically set to the WGS84 Geocentric.

For local ellipsoid GRS 80 is used and for projection - Lambert conformal conic projection with 2 standard parallels.

There are many factors that affect scanning. These factors include high speed, increased traffic, vegetation, and more. Because of them, it is impossible to scan the object in its entirety and white spots appear in the point cloud. The model created from 10 separate records is relatively well-balanced, although the geo-referencing is not very good due to the heavy traffic on Evlogi and Hristo Georgievi Blvd, the high vegetation and the densely built up area.

Figures 18 and 19 show the white spots that appear after filtering the point cloud. High vegetation, which is also a big problem, is filtered.

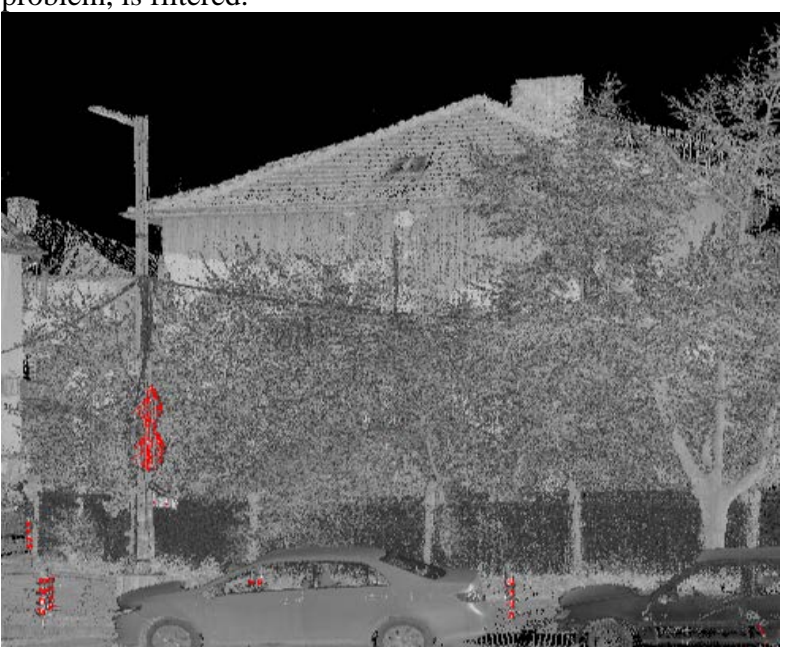

Figure 18: Before filtering 


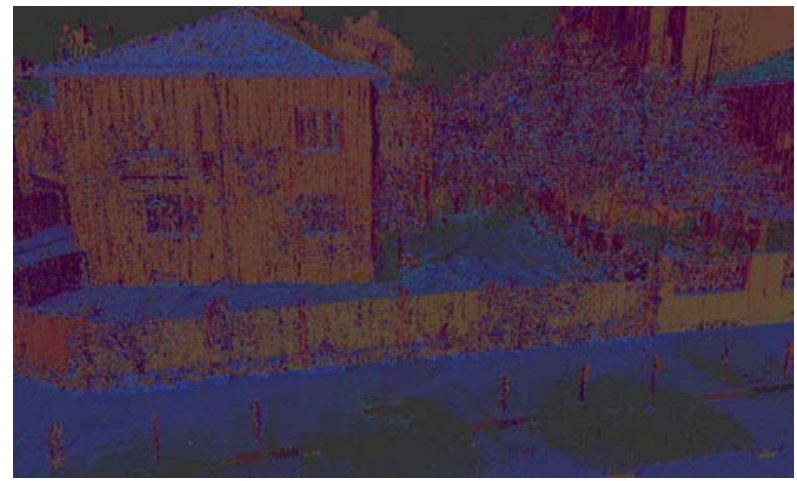

Figure 19: After filtering

Figures 20, 21 and 22 show the final scan result. It is a coordinated point cloud that have been processed with Autodesk ReCap and Civil 3D.

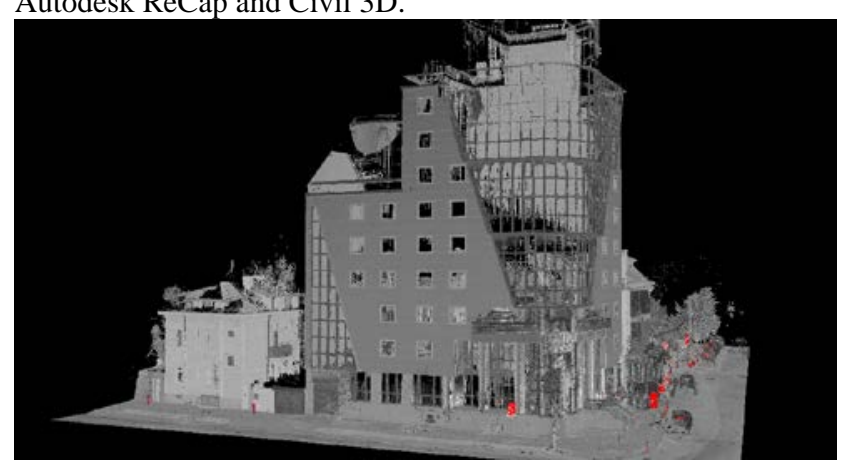

Figure 20: Building in a parcel 68134.902.236 - point cloud

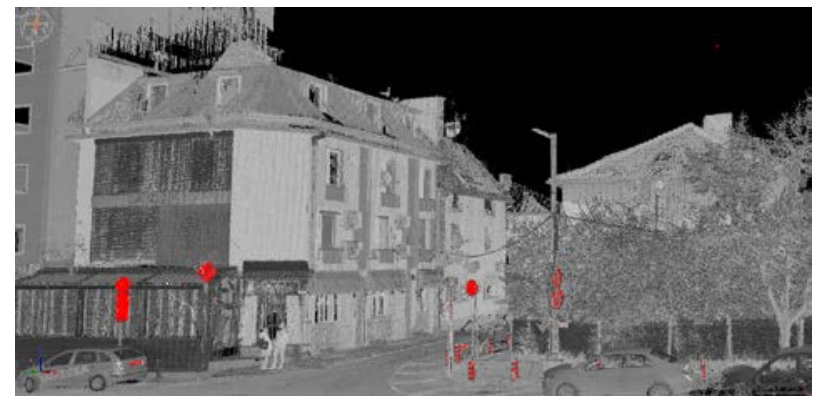

Figure 21: Buildings 3 and 4 in a parcel 68134.902.235 - point cloud

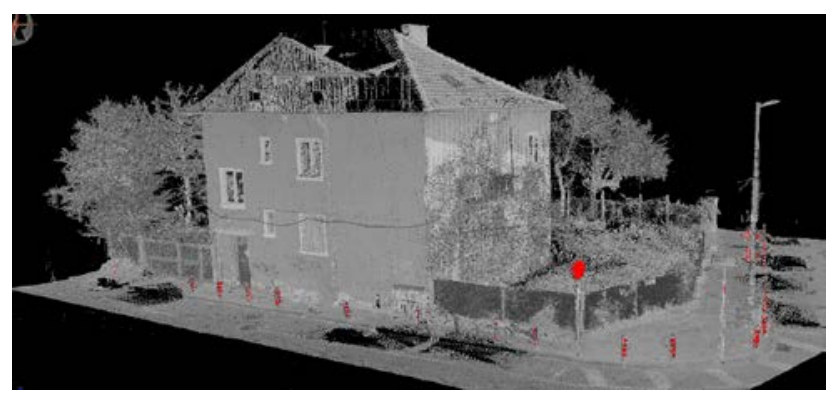

Figure 22: Building in a parcel 68134.902.248 - point cloud Control measurements were also made of the point cloud.

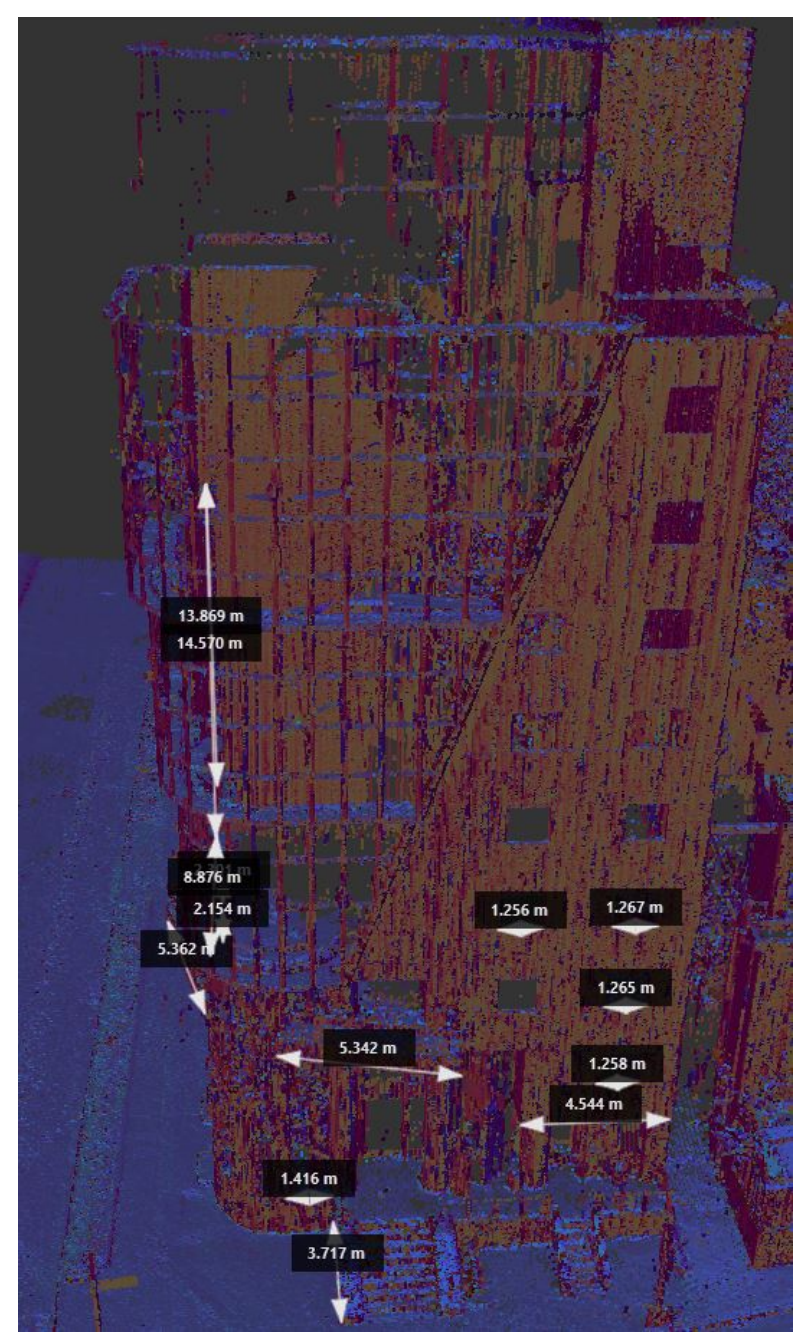

Figure 23: Control measurements

\subsection{Quality control}

During fieldwork, control distances on buildings were directly measured. After constructing the 3D models by a total station, and a mobile laser scanner, control distances in the graphical environment of Civil 3D and Autodesk ReCap were also measured. The distances are measured on clearly distinguishable objects from the facades of the buildings such as window widths, wall lengths, plinth height, etc.

37 control distances were measured for model evaluation. The differences are formed:

$\delta S=S-S_{0}$

where $S_{0}$ is the distance between 2 points from the model $\mathrm{S}$ - the same control distance.

The root mean square error is calculated and a check for gross errors was made.

$m_{x}=\sqrt{\frac{\sum_{1}^{n} \partial S_{i}^{2}}{n}}$

The results are given at table 1 . 


\begin{tabular}{|c|c|c|c|c|c|}
\hline № & $\begin{array}{c}\text { Directly } \\
\text { measured } \\
\text { distances, } \\
{[\mathrm{m}](1)}\end{array}$ & $\begin{array}{c}\text { 3D } \\
\text { model } \\
- \text { total } \\
\text { station } \\
\text {, [m] } \\
\text { (2) }\end{array}$ & $\begin{array}{c}\text { 3D } \\
\text { model } \\
- \\
\text { MLS, } \\
{[\mathrm{m}]} \\
(3)\end{array}$ & $\begin{array}{c}\text { Diference, } \\
{[\mathrm{cm}]} \\
(2)-(1)\end{array}$ & $\begin{array}{c}\text { Diference, } \\
\text { [cm] } \\
\text { (3)-(1) }\end{array}$ \\
\hline 1 & 4.554 & 4.554 & 4.544 & 0.0 & -1.0 \\
\hline 2 & 5.319 & 5.320 & 5.342 & 0.1 & 2.3 \\
\hline 3 & 5.343 & 5.343 & 5.362 & 0.0 & 1.9 \\
\hline 4 & 2.390 & 2.409 & 2.300 & 1.9 & - \\
\hline 5 & 1.450 & 1.445 & 1.416 & -0.5 & -3.4 \\
\hline 6 & 9.057 & 9.056 & 8.876 & -0.1 & - \\
\hline 7 & 0.470 & 0.468 & 0.469 & -0.2 & $\begin{array}{l}-0.1 \\
\end{array}$ \\
\hline 8 & 0.460 & 0.452 & 0.455 & -0.8 & -0.5 \\
\hline 9 & 0.480 & 0.480 & 0.483 & 0.0 & 0.3 \\
\hline 10 & 1.221 & 1.222 & 1.226 & 0.1 & 0.5 \\
\hline 11 & 1.260 & 1.261 & 1.259 & 0.1 & -0.1 \\
\hline 12 & 8.477 & 8.469 & 8.473 & -0.8 & -0.4 \\
\hline 13 & 1.587 & 1.572 & 1.609 & -1.5 & 2.2 \\
\hline 14 & 1.584 & 1.591 & 1.571 & 0.7 & -1.3 \\
\hline 15 & 1.563 & 1.564 & 1.570 & 0.1 & 0.7 \\
\hline 16 & 1.550 & 1.599 & 1.547 & - & -0.3 \\
\hline 17 & 1.580 & 1.619 & 1.592 & - & 1.2 \\
\hline 18 & 14.550 & 14.552 & 14.570 & 0.2 & 2.0 \\
\hline 19 & 13.857 & 13.867 & 13.869 & 1.0 & 1.2 \\
\hline 20 & 1.055 & 1.057 & 1.067 & 0.2 & 1.2 \\
\hline 21 & 0.477 & 0.491 & 0.480 & 1.4 & 0.3 \\
\hline 22 & 1.273 & 1.286 & 1.240 & 1.3 & -3.3 \\
\hline 23 & 1.211 & 1.230 & 1.202 & 1.9 & $\begin{array}{l}-0.9 \\
\end{array}$ \\
\hline 24 & 11.284 & 11.289 & 11.311 & 0.5 & 2.7 \\
\hline 25 & 1.241 & 1.261 & 1.234 & 2.0 & -0.7 \\
\hline 26 & 1.560 & 1.560 & 1.565 & 0.0 & 0.5 \\
\hline 27 & 2.550 & 2.559 & 2.561 & 0.9 & 1.1 \\
\hline 28 & 1.705 & 1.734 & 1.703 & 2.9 & -0.2 \\
\hline 29 & 1.190 & 1.204 & 1.185 & 1.4 & -0.5 \\
\hline 30 & 1.332 & 1.328 & 1.334 & -0.4 & 0.2 \\
\hline 31 & 3.720 & 3.722 & 3.717 & 0.2 & -0.3 \\
\hline 32 & 1.240 & 1.243 & 1.256 & 0.3 & 1.6 \\
\hline 33 & 1.248 & 1.254 & 1.267 & 0.6 & 1.9 \\
\hline 34 & 1.256 & 1.252 & 1.265 & -0.4 & 0.9 \\
\hline 35 & 1.251 & 1.256 & 1.258 & 0.5 & 0.7 \\
\hline 36 & 0.950 & 0.955 & 0.927 & 0.5 & -2.3 \\
\hline 37 & 1.569 & 1.570 & 1.570 & 0.1 & 0.1 \\
\hline \multirow{3}{*}{\multicolumn{4}{|c|}{$\begin{array}{r}M_{x}= \\
m_{x}= \\
3 m_{x}=\end{array}$}} & 0.4 & 0.2 \\
\hline & & & & 1.0 & 1.4 \\
\hline & & & & 2.9 & 4.3 \\
\hline
\end{tabular}

Table 1. Quality control

\section{CONCLUSION}

Mobile laser scanning data provides a large amount of data for captured objects. Spatial data are collected very quickly and at the same time the method provides high accuracy of measurements.

Classical measurement methods are related to data collection using a total station and/or GNSS. The process of work is slowed down because it involves additional exploration of the existing control network and the possible laying of additional points needed for the geodetic survey. On the other hand, the detail points have to be measured one at a time during the surveying, while in mobile laser scanning the speed at which the details are measured can reach up to $1,000,000$ points per second.
Of course, the investment in a mobile scanner is many times greater than that of a total station. To justify, a large amount of work is needed to be done quickly. This is the advantage of this type of scanning system.

However, they also have their disadvantages:

- The reflectivity of the surfaces has a great influence

- In a highly urbanized area (such as the case study) it is possible that not very good results can be obtained. The possible reasons are different - such as the lack of enough satellites, high vegetation, heavy traffic, high buildings, lack of access to the internal borders of the parcels.

Nevertheless, laser scanning is the fastest method of collecting spatial data - high range, high accuracy, high degree of detail of captured objects.

\section{ACKNOWLEDGEMENTS}

The authors express their gratitude to GeoTech Engineering LTD. Without their kindly support with mobile laser scanner, the Alexander's master thesis and this paper would not be done.

\section{REFERENCES}

Alexandrov B., V. Valchinov, 2012, Quantitative control of raw materials by land surveying, Geomedia, vol. 6

Antova, G., 2006, Rapid precise mapping with 3D laser scanning, Annual of UACEG, Sofia, Bulgaria

Coşarcă C., A. Jocea, A. Savu, 2009, Analysis of error sources in Terrestrial Laser Scanning

Dzhenkova R. , 2019, Investigation on Terrestrial Laser Scanning, Master Thesis, UACEG, Sofia

Fröhlich, C. and Mettenleiter, M., 2004, Terrestrial laser scanning - New perspectives in 3D surveying. Laser scanners for forest and landscape assessment., International Archives of Photogrammetry, Remote Sensing and Spatial Information Sciences, 36(Part 8, W2):7-13

Ivanov R., 2020, Engineering Surveying And Deformation Surveys, ISBN 978-954-12-0272-2, Monograph, Sofia

Mills, J. and Barber, D., 2003, The collection and archiving of point cloud data obtained by terrestrial laser scanning or other methods, In An Addendum to the Metric Survey Specifications for English Heritage

Pavlov P., 1999, Analysis and quality control of cadastral information obtained by surveying and digitalisation. Geodesy, cartography and land management. Sofia, ISSN 0324-1610, Issue 5-6

\section{Revised January 2020}

